\title{
Fates of retained hepatic segment IV and its prognostic impact in adult split liver transplantation using an extended right liver graft
}

\author{
Sung-Min Kim, Shin Hwang, Chul-Soo Ahn, Ki-Hun Kim, Deok-Bog Moon, Tae-Yong Ha, Gi-Won Song, Dong-Hwan Jung, \\ Gil-Chun Park, Sung-Gyu Lee
}

Department of Surgery, Asan Medical Center, University of Ulsan College of Medicine, Seoul, Korea

Background: When splitting a liver for adult and pediatric graft recipients, the retained left medial section (S4) will undergo ischemic necrosis and the right trisection graft becomes an extended right liver (ERL) graft. We investigated the fates of the retained S4 and its prognostic impact in adult split liver transplantation (SLT) using an ERL graft.

Methods: This was a retrospective analysis of 25 adult SLT recipients who received split ERL grafts.

Results: The mean model for end-stage liver disease (MELD) score was $27.3 \pm 10.9$ and graft-recipient weight ratio (GRWR) was $1.98 \pm 0.44$. The mean donor age was $26.5 \pm 7.7$ years. The split ERL graft weight was $1,181.5 \pm 252.8 \mathrm{~g}$, which resulted in a mean GRWR of $1.98 \pm 0.44$. Computed tomography of the retained S4 parenchyma revealed small ischemic necrosis in 16 patients (64\%) and large ischemic necrosis in the remaining nine patients (36\%). No S4-associated biliary complications were developed. The peak liver enzyme levels were higher in the large $S 4$ ischemic necrosis group $(P \leq 0.002)$. The mean GRWR was $1.87 \pm 0.43$ in the nine patients with large ischemic necrosis and $2.10 \pm 0.44$ in the 15 cases with small ischemic necrosis $(P=0.28)$. The retained S4 parenchyma showed gradual atrophy on follow-up imaging studies. The amount of S4 ischemic necrosis was not associated with graft $(P=0.59)$ or patient $(P=0.24)$ survival. A MELD score $>30$ and pretransplant ventilator support were associated with inferior outcomes.

Conclusions: The amount of ischemic necrosis of the retained S4 parenchyma is not a negative prognostic indicator in adult SLT recipients, likely due to sufficiently large GRWR. However, a high MELD score ( $>30)$ and pretransplant ventilator support are closely associated with inferior outcomes in these cases. Therefore, careful selection of donors and recipients is essential to improve the outcomes of adult SLT.

Corresponding author: Shin Hwang

E-mail: shwang@amc.seoul.kr

\footnotetext{
(c) The Korean Society for Transplantation

This is an Open Access article distributed under the terms of the Creative Commons Attribution Non-Commercial License (http://creativecommons.org/licenses/by-nc/4.0/) which permits unrestricted non-commercial use, distribution, and reproduction in any medium, provided the original work is properly cited.
} 